\title{
A prognostic index based on a fourteen long non-coding RNA signature to predict the recurrence-free survival for muscle- invasive bladder cancer patients
}

Xiaolong Zhang ${ }^{1,2,3 \dagger}$, Meng Zhang ${ }^{3,4 \dagger}$, Xuanping Zhang ${ }^{1}$, Xiaoyan Zhu ${ }^{1}$ and Jiayin Wang ${ }^{{ }^{*}}$

From 5th China Health Information Processing Conference

Guangzhou, China. 22-24 November 2019

\begin{abstract}
Background: Bladder cancer (BC) is regarded as one of the most fatal cancer around the world. Nevertheless, there still lack of sufficient markers to predict the prognosis of BC patients. Herein, we aim to establish a prognosis predicting signature based on long-noncoding RNA (InCRNA) for the invasive BC patients.

Methods: The IncRNA expression profile was downloaded from The Cancer Genome Atlas (TCGA) database, along with the correlated clinicopathological information. The univariate Cox regression test was employed to screen out the recurrence-free survival (RFS)-related IncRNAs. Then, the LASSO method was conducted to construct the signature based on these RFS-related IncRNA candidates. Genes correlated with these fourteen IncRNAs were extracted from the mRNA expression profile, with the Pearson correlation coefficient $>0.60$ or $<-0.40$.

Subsequently, the Proteomap pathway enrichment analyses were conducted to classify the function of these correlated genes. Furthermore, the multivariate analyses were executed to reveal the independent role of the proposed signature with the clinicopathological features.

(Continued on next page)
\end{abstract}

\footnotetext{
* Correspondence: wangjiayin@mail.xjtu.edu.cn

${ }^{+}$Xiaolong Zhang and Meng Zhang contributed equally to this work.

'School of Computer Science and Technology, Shaanxi Engineering Research Center of Medical and Health Big Data, Xi'an Jiaotong University, Xi'an, China

Full list of author information is available at the end of the article
}

(c) The Author(s). 2020 Open Access This article is licensed under a Creative Commons Attribution 4.0 International License, which permits use, sharing, adaptation, distribution and reproduction in any medium or format, as long as you give appropriate credit to the original author(s) and the source, provide a link to the Creative Commons licence, and indicate if changes were made. The images or other third party material in this article are included in the article's Creative Commons licence, unless indicated otherwise in a credit line to the material. If material is not included in the article's Creative Commons licence and your intended use is not permitted by statutory regulation or exceeds the permitted use, you will need to obtain permission directly from the copyright holder. To view a copy of this licence, visit http://creativecommons.org/licenses/by/4.0/ The Creative Commons Public Domain Dedication waiver (http://creativecommons.org/publicdomain/zero/1.0/) applies to the data made available in this article, unless otherwise stated in a credit line to the data. 
(Continued from previous page)

Results: We established an IncRNA-based RFS predicting signature by the LASSO Cox regression test, and proved its usage and stability on both the training and validation cohorts by the Kaplan-Meier and receiver operating characteristic (ROC) curves. Notably, the multivariate Cox regression analysis found that our classifier was an independent indicator for muscle-invasive BC patients rather than sex, age and tumor grade, with higher predictive value than the existing ones. Besides, we did the pathway analyses for these genes that highly correlated with the proposed fourteen IncRNAs, as well as the differentially expressed genes (DEGs) derived from the high-risk vs. lowrisk groups, and the recurrence vs. non-recurrence groups, respectively. Notably, these results were consistent, and these genes were mostly enriched in the transcription factors, G protein-coupled receptors, MAPK signaling pathways, which were proved significantly associated with tumor progression and drug resistance.

Conclusions: Our results suggested that the fourteen-IncRNA-based RFS predicting signature is an independent indicator for BC patients. Further prospective studies with more samples are needed to verify our findings.

Keywords: Clinical decision-supporting, Muscle-invasive bladder cancer, Long non-coding RNA, Prognosis signature

\section{Background}

Bladder cancer (BC) is known as the ninth most frequent malignancy around the world. Because of high rate of recurrence, it has been one of the most expensive solid cancer once the patients seed for continued surveillance [1]. Further, the high recurrence rate of $\mathrm{BC}$ is partly owning to the insufficient number of prognosisrelated biomarkers. Thus, to find out more favorable biomarkers for early detection and prognosis prediction of bladder cancer is becoming more critical.

The long non-coding RNAs (lncRNAs) are characterized as RNA transcripts $>200$ bases, but they cannot translate into proteins [2, 3]. Currently, plenty of studies have indicated that lncRNAs participate in diverse biological processes, such as cell proliferation [4], differentiation [5], chromatin modification, and so on [6]. The lncRNAs have been revealed playing critical roles in tumorigenesis and progression. Referring to previous studies, a series of lncRNAs, such as MALAT1, TUG1, $\mathrm{H} 19$, played a critical role in predicting the prognosis, the risk of metastasis [7-11], as well as drug treatment resistance [12]. Mechanisms studies found the function of lncRNA is partly depended on its location. For example, the IncRNA-HOTAIR, located in the nuclear, regulates the gene expression through recruiting the chromatin modifiers on the promoter region of transcriptional factors, enhancing their transcriptional activities. For these lncRNAs located in the cytoplasm, regulates the protein expression mostly through posttranscription, such as influencing the protein stability.

Besides, the lncRNAs could also serve as competing endogenous RNAs to influence the expression of targeted genes by competing with the microRNAs (miRNAs). In addition to the functional role of lncRNAs, many other studies have highlighted their role in predicting the prognosis of cancer patients. For example, Yang et al. [13] identified a six-lncRNA-based signature, which can predict the recurrence risk of ovarian cancer.
In Song et al.'s [14] work, they established a lncRNAbased signature that provides the prognostic value for outcomes of gastric cancer patients. Similar studies were also conducted for thyroid papillary carcinoma [15], pancreatic cancer [16] and esophageal squamous cell carcinoma [17], and revealed satisfied outcomes. Although few studies have tried to construct gene-based signature for bladder cancer patients, there is no study specifically focus on muscle-invasive BC patients, who mostly have poor survival outcome than these patients with in situ tumors.

Here, we performed a systematic screening of lncRNAs that related to the recurrence-free survival (RFS) of muscle-invasive BC patients, and established a fourteen-lncRNA-based signature. Our study provides the tool for clinicians to develop the personalized medicine for muscle-invasive $\mathrm{BC}$ patients.

\section{Methods}

\section{Bladder cancer datasets and patient information}

Clinical information, particularly for RFS (status and follow-up time), and the RNA expression profile for lncRNAs in invasive $\mathrm{BC}$ tissues were directly download from TCGA database (https://cancergenome.nih.gov/). A total of 320 patients were diagnosed as muscle-invasive $\mathrm{BC}$, and then they were divided into the training and validating groups (224 vs. 96), randomly.

\section{Signature construction and risk stratification}

The lncRNAs were subjected to the univariable Cox regression analysis to find out IncRNAs that correlated with the RFS of muscle-invasive BC patients. Then, the LASSO Cox regression analysis was conducted to establish the risk signature based on these RFS-related lncRNA candidates. The risk value of each patient was depended on the expression of lncRNAs, and their matched co-efficient. The formula of our signature was presented as below: 


$$
\begin{aligned}
\text { Risk score }= & \beta_{\text {lncRNA1 }} \times \operatorname{expr}_{\mathrm{lncRNA1}}+\beta_{\mathrm{lncRNA} 2} \\
& \times \operatorname{expr} r_{\mathrm{IncRNA} 2}+\ldots+\beta_{\mathrm{lncRNAn}} \times \beta_{\mathrm{lncRNAn}}
\end{aligned}
$$

The patients in both the training and validation cohorts were ranked by the risk scores, and then they were classified into high- and low-risk according to the median risk score derived from the training cohort.

\section{Kaplan-Meier (K-M) and receiving operating curve (ROC)}

The K-M analysis was applied to find out the RFS difference between the low- and high-risk groups, and the ROC curve was used to determine the prognostic value of our signature in both the training and validation cohorts. Hence, the multivariate analyses were executed to determine the independent effect of our signature with clinicopathological features (such as sex, age, tumor grade and tumor stage). All these analyses were conducted based on R software (version 3.5.2) with the following R packages: 'glmnet', 'survivalROC' and 'ggplot2'. Besides, the $P$-value less than 0.05 was considered as statistically significant.

\section{Differential expression and functional analysis}

Differential expression analysis was performed by DESeq2 [18], and differentially expressed genes (DEGs) were defined as fold change more than 1 and adjusted $p$-value less than 0.05 . Functional categories of genes were analyzed using Proteomap (https://www.proteomaps.net/).

\section{Results}

\section{Signature establishment}

The muscle-invasive BC patients $(n=320)$ were divided into the training cohort $(n=224)$ and a validation cohort $(n=96)$, randomly. Subsequently, the univariate Cox regression test was executed to verify the RFS-related
lncRNAs (Fig. S1, P-value less than 0.05). The predicting signature was constructed based on the training cohort with the LASSO method. As a result, the formula of the signature was defined as below (Fig. 1): Risk score $=$ (0.052146 x expression value of C21orf34) - $(0.20576 \times$ expression value of $\mathrm{C} 22$ orf45 $)+(0.164629 \times$ expression value of C4orf12 $)+(0.136011 \times$ expression value of C7orf13 $)+(0.057299 \times$ expression value of CACNA2D1 $)$ - (0.10285 x expression value of HCG4P6) - (0.18816 × expression value of INE2) - $(0.14616 \times$ expression value of LOC115110 $)-(0.12243 \times$ expression value of LOC283663 $)-(0.09584 \times$ expression value of LOC554202 $)$ - $(0.09684 \times$ expression value of SNHG10 $)+(0.095918 \times$ expression value of SOX2OT $)+$ $(0.123121 \times$ expression value of STL $)+(0.207295 \times$ expression value of SYS1-DBNDD2). Referring to the formula, the risk score for the patients in both the training and validation cohorts was generated. Patients were ranked according to their risk scores, and referring to the median risk score of the training cohort as the cutoff point, these patients were assigned into low- and highrisk groups.

\section{Survival and ROC analyses}

We managed the K-M analysis with the log-rank test to compare the difference in recurrence rate between the low- and high-risk groups. As shown in Fig. 2a, we found that the patients with the high-risk scores tended to experience a higher recurrence rate than for those with low-risk scores in the training cohort $(P<0.0001)$. The similar results also presented in the validation set $(P=0.0022$; Fig. $2 b)$. Furthermore, the ROC curves were applied to determine the predictive values of our signature. The results showed that the AUC value of the training cohort was $0.796(95 \% \mathrm{CI}$ : $71.6-87.7)$, while it was $0.748(95 \% \mathrm{CI}, 61.8-87.7)$ in
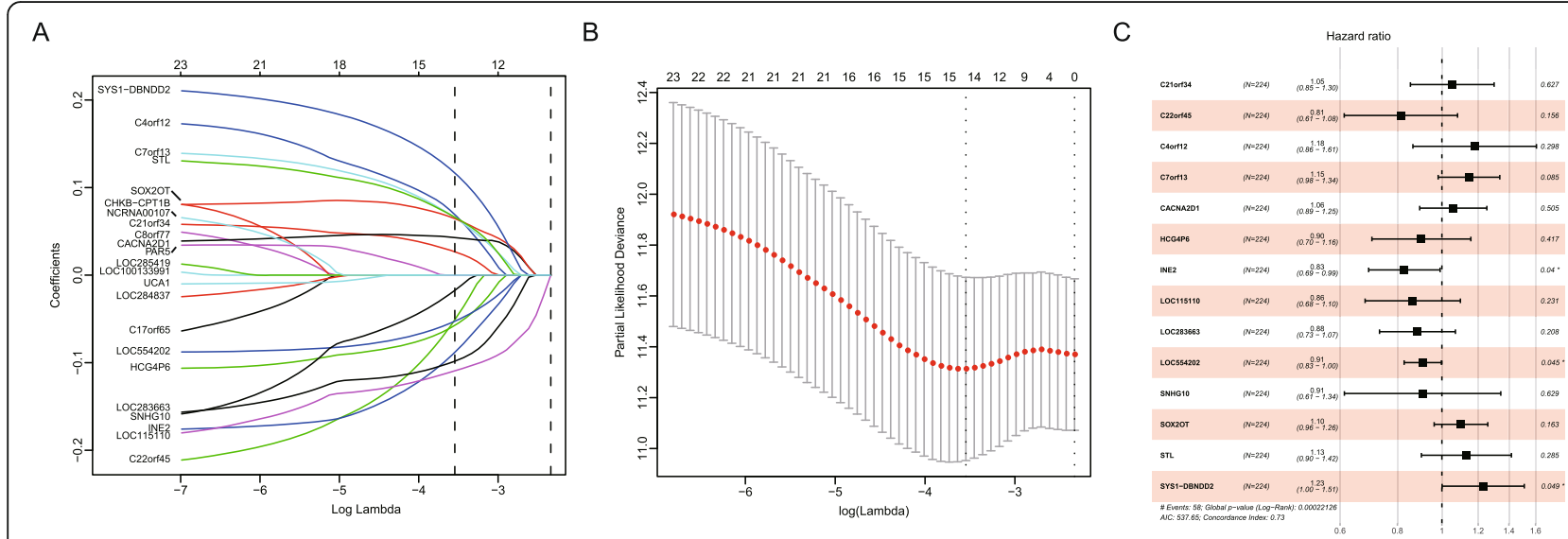

Fig. 1 Used the LASSO Cox regression test to construct the IncRNA-based recurrence-free survival predicting classifier. a. LASSO coefficient profiles of the fourteen features for RFS. b. Tuning parameter (lamda) selection in the LASSO model used 10-fold cross-validation via minimum criteria for RFS. c. Forest plot showing multivariate Cox regression analysis of the effect of different IncRNAs on patient RFS 
A

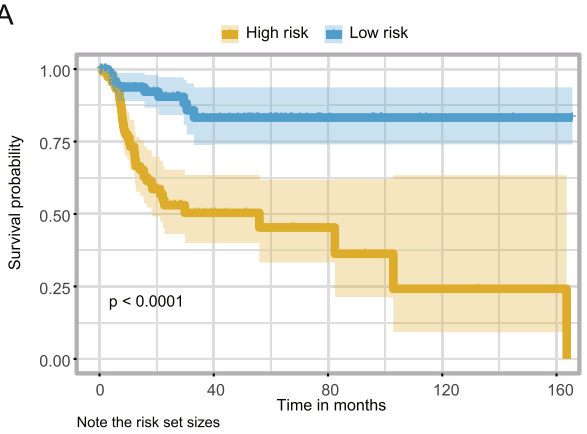
Note the risk set sizes

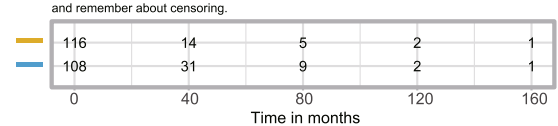

C

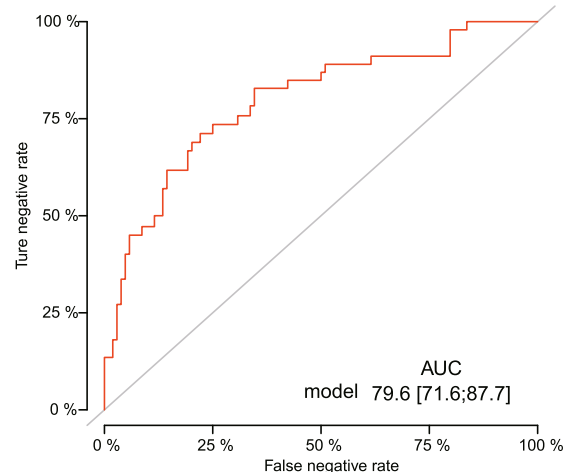

B

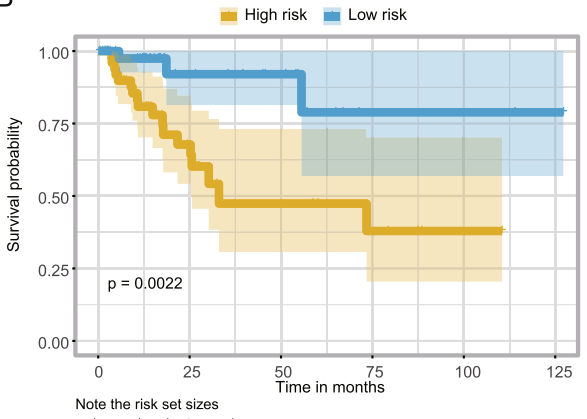

Note the risk set sizes

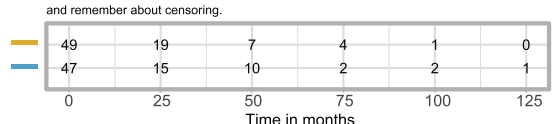

D

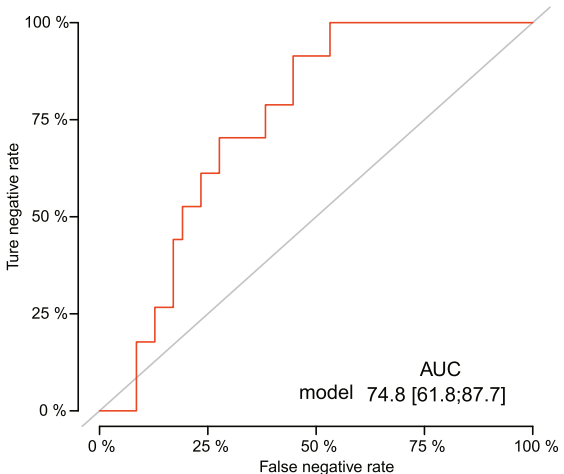

Fig. 2 Fourteen-IncRNA-based RFS predicting classifier performance in MIBC. a and $\mathbf{b}$. Kaplan-Meier curve of the low- and high-risk groups verified by the fourteen-IncRNA-based overall survival predicting classifier in the training and validation set, respectively. $\mathbf{c}$ and $\mathbf{d}$. ROC curve of the low- and high-risk sets verified by the fourteen-IncRNA-based recurrence-free survival predicting classifier in the training and validation set, respectively

A

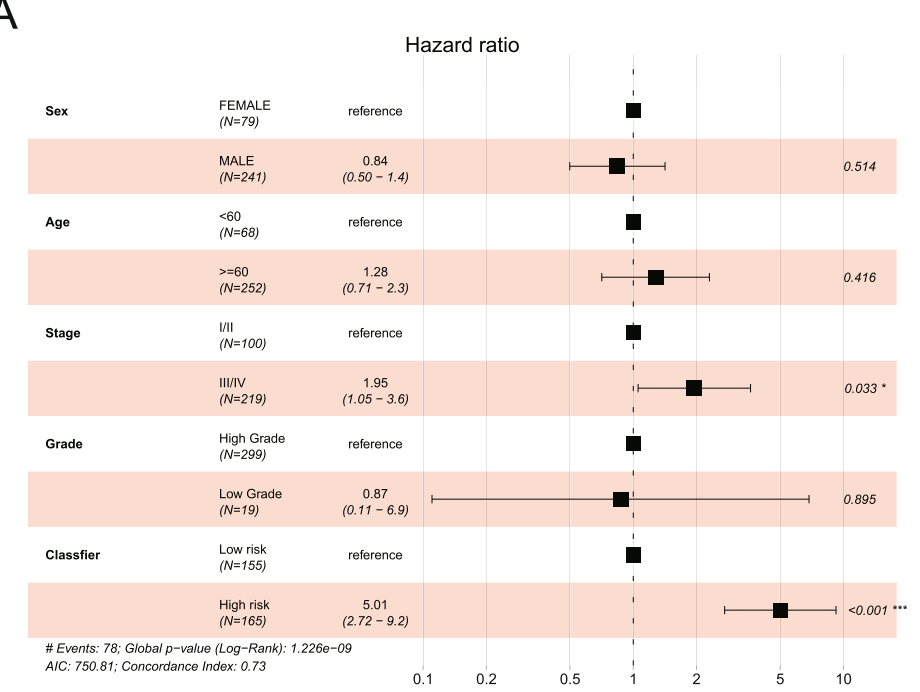

B

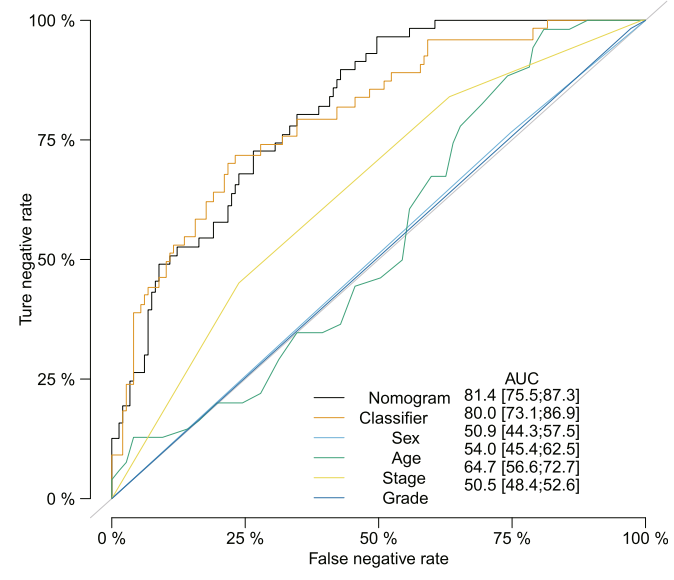

Fig. 3 The comparison between the fourteen-IncRNA-based RFS classifier and clinicopathological features in all MIBC samples. a. Univariate Cox proportional-hazards regression analysis results of fourteen-IncRNA-based classifier and clinicopathological features, respectively. $\mathbf{b}$. ROC curve of low- and high-risk sets verified by the fourteen-IncRNA-based classifier and clinicopathological features, respectively 
the validation cohort (Fig. 2c and d), indicating that our signature had a moderate predictive accuracy and reliability in evaluating the prognosis for muscleinvasive $\mathrm{BC}$ patients.

\section{Multivariate analyses}

We performed the multivariable Cox regression analysis to adjust the clinical variables (age, sex, tumor stage and grade), and the results showed our fourteen-lncRNAbased RFS and tumor stage (stage III + IV) remained to be the independent prognostic factors for muscleinvasive BC patients' RFS in the overall dataset (Fig. 3a). The HR (HR $=5.01,95 \% \mathrm{CI}$ : 2.72-9.2) for the integrated lncRNA signature was greater than tumor stage $(\mathrm{HR}=$ 1.95, 95\%CI: 1.05-3.6), and it implied that the signature had superior performance compared with the tumor stage.

Besides, we also compared our classifier with other clinicopathological features (age, sex, tumor grade, and tumor stage). We found the signature showed a high AUC value of 0.80 , which was better than any of the other features (Fig. 3b). The nomogram was performed to determine the synthesis effects by combining our signature with clinicopathological features, and the results indicated that the nomogram had the best predicting values (AUC $=81.4,95 \% \mathrm{CI}$ : 75.5-87.3).

In addition, the subgroup analyses were executed referring to the clinicopathological features (age, sex, tumor grade, and tumor stage), and the results showed that the fourteen-lncRNA-based RFS signature still could classify the risk stratification in spite of age $(<60$, and $>60$ ), sex (male, and female) (Fig. 4a-d). Further, our signature was also effective for these patients with higher stage (III/IV; $P<0.0001)$ and grade $(\mathrm{T} 3+\mathrm{T} 4 ; P<0.0001)$ (Fig. 4e-h).

\section{Identification of eight IncRNA signature associated biological pathways and processes}

To reveal the underlying mechanisms that how these fourteen lncRNAs influenced tumor progression. We extracted the mRNA expression profile from the TCGA database. For these genes highly correlated with the fourteen lncRNAs were enrolled (Pearson correlation coefficient $>0.5$ or $<-0.5$ ). Then, the proteomap pathway analysis was performed to classify their functions. The results found that these genes were mostly enriched in Transcription factors, Peptidases, Ion channels, G protein-coupled receptors, Glycan metabolism, MAPK signaling, Wnt signaling, and ErbB signaling pathways, which were proved highly associated with tumor initiation, progression and drug resistance (Fig. 5a). Notably, the DEGs were also obtained by comparing the recurrence set vs. non-recurrence set, and high-risk vs. lowrisk groups, respectively, with similar results obtained (Fig. $5 \mathrm{~b}$ and $\mathrm{c}$ ). All these results proved the application value of our signature in predicting the RFS prognosis

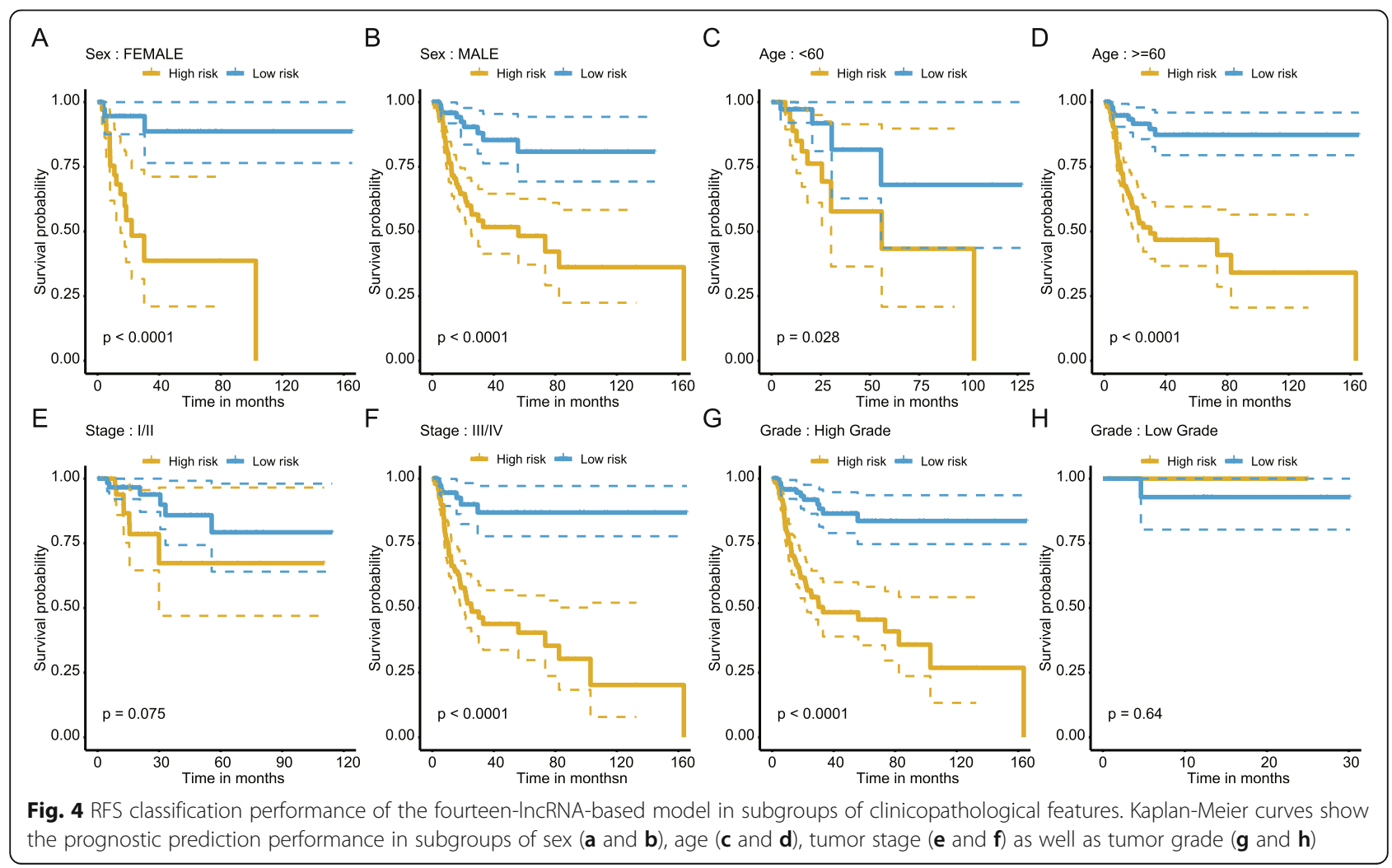



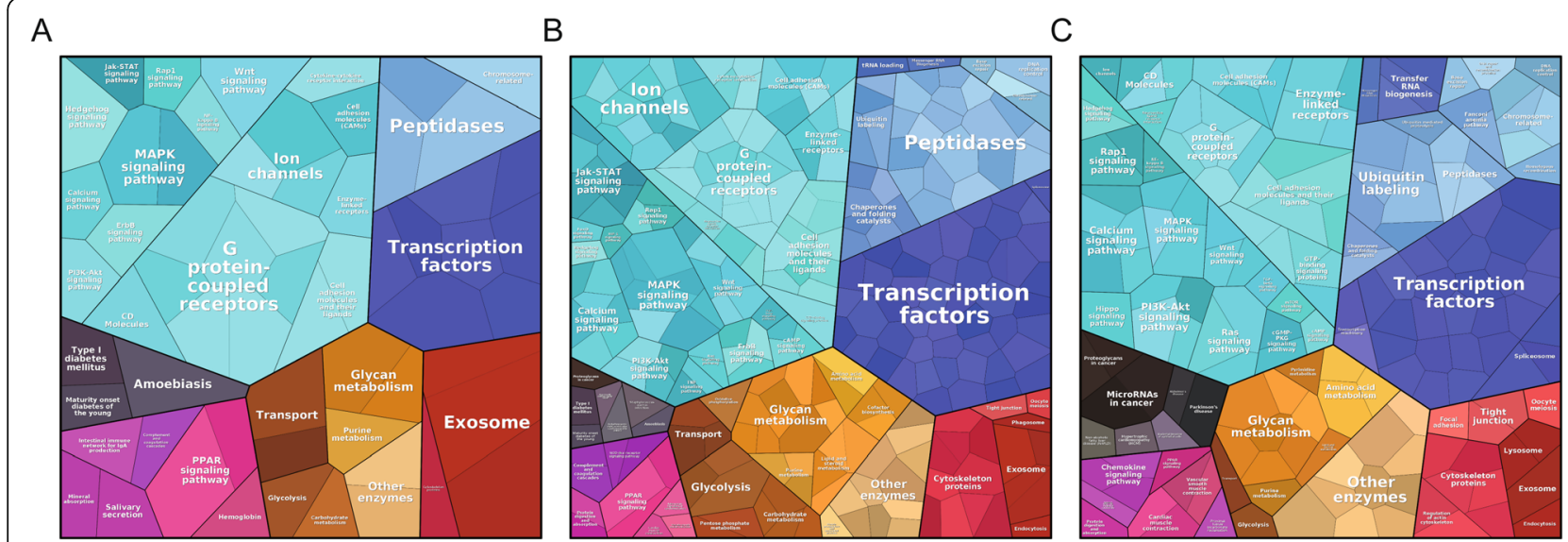

Fig. 5 Gene functional categories showed by Proteomap. a. Functional category of genes which expressed highly related IncRNA markers. $\mathbf{b}$. Functional category of genes differentially expressed between recurrent and non-recurrent MIBC samples. $\mathbf{c}$. Functional category of genes differentially expressed between predicted high- and low-risk sample groups

for muscle-invasive $\mathrm{BC}$ patients, and provided the potential mechanisms for how these lncRNAs influenced the $\mathrm{BC}$ progression.

\section{Discussion}

The disease progression of muscle-invasive $\mathrm{BC}$ patients is dependent on many risk factors including phenotypes and genotypes. However, clinical criteria such as age, gender, pathological TNM stage and tumor grade may not reflect the entire biology of muscle-invasive $\mathrm{BC}$. Here, we investigated the efficacy of the 14-IncRNAbased gene signature to predict the RFS of muscleinvasive $\mathrm{BC}$ patients. Despite previously developed gene signature-based prognostic models, it is still valuable to update new models to improve the management of muscle-invasive $\mathrm{BC}$. An effective gene signature could guide patient counseling and help people to identify candidates who need more aggressive management. We demonstrated that this model has more prediction power than independent traditional clinical features. Although it is lack of novelty and function work in our study and our results require further investigation of the efficacy of the 14-IncRNA-based signature panel in patients, this panel could be extremely beneficial to identify patients at elevated risk of recurrence that may require adjuvant therapy.

We identified a set of 14 lncRNAs that showed differential expressions between high-and low-risk cancer patients included in the data sets (Fig. S2). Such differentiations signified their potential roles in carcinogenesis. Recent researches has found that IncRNA LOC554202 is significantly downregulated in bladder cancer tissues compared with adjacent noncancerous tissues, and lncRNA LOC554202 expression level in bladder cancer patients was negatively associated with advanced TNM stage [19]. SNHG10 is known to be over-expressed in hepatocellular carcinoma, and we found it facilitates hepatocarcinogenesis and metastasis [20]. SOX2 overlapping transcript mainly play crucial role in tumor initiation and/or progression as well as regulating pluripotent state of stem cells [21]. CACN A2D1 is most the most extensively investigated and validated of these markers. A retrospective study showed that positive expression of Cacna2d1 was significantly associated with advanced FIGO stage $(P<0.001)$, histological subtype $(P=0.017)$ and tumor differentiation $(P=0.015)$ [22]. Data coming from Sui et al.'s research [23] have confirmed that radio-resistance of non-small cell lung cancer induced by CaCna2D1. The roles of the rest of the lncRNA genes identified in bladder cancer remain unclear.

\section{Conclusion}

By applying public TCGA data, we successfully built and validated a RFS prediction model of muscle-invasive $B C$ based on a novel 14-lncRNA signature. Comparing with independent clinical features, this model has more efficiency to predict RFS of muscle-invasive $\mathrm{BC}$. The model may help facilitate doctor-patient consultations, guide muscle-invasive $\mathrm{BC}$ treatment strategy and eventually benefit patients.

\section{Supplementary information}

Supplementary information accompanies this paper at https://doi.org/10. 1186/s12911-020-1115-2.

Additional file 1: Supplementary Figure 1. Kaplan-Meier curves show 23 IncRNAs which significantly related to recurrence-free survival of MIBC in the training data.

Additional file 2: Supplementary Figure 2. Expression level of the fourteen prognostic IncRNA markers in high- and low-risk MIBC groups, respectively. 


\section{Abbreviations}

BC: Bladder cancer; DEGs: Differentially expressed genes; FDR: False discovery rate; K-M: Kaplan-Meier; IncRNA: long-noncoding RNA; miRNAs: microRNAs; RFS: Recurrence-free survival; ROC: Receiver operating characteristic; TCGA: The Cancer Genome Atlas

\section{Acknowledgements}

The authors would like to thank the conference organizers of the China Conference on Health Information Processing (CHIP 2019). We also would like to thank the reviewers for their valuable comments and suggestions, which guide us to improve the work and manuscript.

\section{About this supplement}

This article has been published as part of BMC Medical Informatics and Decision Making Volume 20 Supplement 3, 2020: Health Information Processing. The full contents of the supplement are available online at https:// bmcmedinformdecismak.biomedcentral.com/articles/supplements/volume-2 0-supplement-3.

\section{Authors' contributions}

In this study, JYW and XLZ designed the study; XLZ and MZ construct the prognosis model and processed the bioinformatics data. $X L Z$ and $M Z$ drafted the manuscript; JYW, XPZ and XYZ revised the final manuscript. All authors have read and approved the final manuscript.

\section{Funding}

The publication costs for this article were funded by China Postdoctoral Science Foundation funded project 2018 M643684. This work was supported by the grants from National Natural Science Foundation of China (31701150, 81702791 and 81802827) and Fundamental Research Funds for the Central Universities (CXTD2017003). The funders have no role in design of the study, data collection, analysis, interpretation, and in writing the manuscript.

\section{Availability of data and materials \\ Not applicable.}

\section{Ethics approval and consent to participate}

Not applicable.

\section{Consent for publication}

Not applicable.

\section{Competing interests}

The authors declare that they have no competing interests.

\section{Author details}

'School of Computer Science and Technology, Shaanxi Engineering Research Center of Medical and Health Big Data, Xi'an Jiaotong University, Xi'an, China. ${ }^{2}$ State Key Laboratory of Oncology in South China, Collaborative Innovation Center for Cancer Medicine, Sun Yat-sen University Cancer Center, Guangzhou, China. ${ }^{3}$ School of Medicine, Shenzhen University, Shenzhen, China. ${ }^{4}$ Department of Urology, The First Affiliated Hospital of Anhui Medical University, Hefei, China.

\section{Published: 9 July 2020}

\section{References}

1. Ilaria L, Michela DM, Tobias K, Shariat SF. Novel biomarkers to predict response and prognosis in localized bladder cancer. J Urol Clin North Am. 2015;42(2):225-33.

2. Zhou M, Guo M, He D, Wang X, Cui Y, Yang H, Hao D, Sun J. A potential signature of eight long non-coding RNAs predicts survival in patients with non-small cell lung cancer. J Transl Med. 2015;13(1):231.

3. Cheetham SW, Gruhl F, Mattick JS, Dinger ME. Long noncoding RNAs and the genetics of cancer. J Br J Cancer. 2013;108(12):2419-25.

4. Liu N, Liu Q, Yang X, Zhang F, Li X, Ma Y, Guan F, Zhao X, Li Z, Zhang L, et al. Hepatitis B virus-upregulated Inc-HUR1 promotes cell proliferation and tumorigenesis by blocking p53 activity. Hepatology. 2018;68(6):2130-44.

5. Lopez-Pajares V, Qu K, Zhang J, Webster DE, Barajas BC, Siprashvili Z, Zarnegar BJ, Boxer LD, Rios EJ, Tao S, et al. A LncRNA-MAF: MAFB transcription factor network regulates epidermal differentiation. Dev Cell. 2015:32(6):693-706.

6. Inma G, Roberto M, Eneritz A, Dittmer TA, Katia G, Tom M, Luco RF. A IncRNA regulates alternative splicing via establishment of a splicing-specific chromatin signature. J Nat Struct Biol M. 2015;22(5):370-6.

7. Gutschner T, Hämmerle M, Eißmann M, Hsu J, Kim Y, Hung G, Revenko A, Arun G, Stentrup M, Groß M. The non-coding RNA MALAT1 is a critical regulator of the metastasis phenotype of lung cancer cells. Cancer Res. 2013:73(3):1180-9.

8. Jin C, Yan B, Lu Q, Lin Y, Ma L. Reciprocal regulation of Hsa-miR-1 and long noncoding RNA MALAT1 promotes triple-negative breast cancer development. Tumour Biol. 2016;37(6):7383-94.

9. Li C, Cui Y, Liu LF, Ren WB, Li QQ, Zhou X, Li YL, Li Y, Bai XY, Zu XB, et al. High Expression of Long Noncoding RNA MALAT1 Indicates a Poor Prognosis and Promotes Clinical Progression and Metastasis in Â Bladder Cancer. Clin Genitourin Cancer. 2018;36(6):570-6.

10. Shi XS, Li J, Yang RH, Zhao GR, Zhou HP, Zeng WX, Zhou M. Correlation of increased MALAT1 expression with pathological features and prognosis in cancer patients: a meta-analysis. 2015;14(4):18808.

11. Zhai H, Chen QJ, Chen BD, Yang YN, Ma YT, Li XM, Liu F, Yu ZX, Xiang Y, Liao W. Long noncoding RNA MALAT1 as a putative biomarker of lymph node metastasis: a meta-analysis. Int J Clin Exp Med. 2015;8(5):7648-54.

12. Tang D, Yang Z, Long F, Luo L, Yang B, Zhu R, Sang X, Cao G. Inhibition of MALAT1 reduces tumor growth and metastasis and promotes drug sensitivity in colorectal cancer. Cell Signal. 2019;57:21-8.

13. Yang K, Hou Y, Li A, Li Z, Wang W, Xie H, Rong Z, Lou G, Li K. Identification of a six-IncRNA signature associated with recurrence of ovarian cancer. Sci Rep. 2017;7(1):752.

14. Song P, Jiang B, Liu Z, Ding J, Liu S, Guan W. A three-IncRNA expression signature associated with the prognosis of gastric cancer patients. Cancer Med. 2017;6(6):1154-64

15. Zhang $H$, Cai $Y$, Zheng L, Zhang Z, Lin X, Jiang N. LncRNA BISPR promotes the progression of thyroid papillary carcinoma by regulating miR-21-5p. Int J Immunopathol Pharmacol. 2018;32:2058738418772652.

16. Shi X, Zhao Y, Zhou M, Pan S, Yu S, Xie Y, Li X, Wang M, Guo X, Qin R. Three-IncRNA signature is a potential prognostic biomarker for pancreatic adenocarcinoma. 2018:9(36):24248-59.

17. Li J, Chen Z, Tian L, Zhou C, He MY, Gao Y, Wang S, Zhou F, Shi S, Feng X, et al. LnCRNA profile study reveals a three-IncRNA signature associated with the survival of patients with oesophageal squamous cell carcinoma. Gut. 2014;63(11):1700-10.

18. Love Ml, Huber W, Anders S. Moderated estimation of fold change and dispersion for RNA-seq data with DESeq2. Genome Biol. 2014;15(12):550.

19. He A, Chen Z, Mei H, Liu Y. Decreased expression of LncRNA MIR31HG in human bladder cancer. Cancer Biomark. 2016;17(2):231-6.

20. Lan T, Yuan K, Yan X, Xu L, Liao H, Hao X, Wang J, Liu H, Chen X, Xie K, et al. LncRNA SNHG10 facilitates Hepatocarcinogenesis and metastasis by modulating its homolog SCARNA13 via a positive feedback loop. Cancer Res. 2019;79(13):3220-34

21. Shahryari A, Rafiee MR, Fouani Y, Oliae NA, Samaei NM, Shafiee M, Semnani S, Vasei M, Mowla SJ. Two novel splice variants of SOX2OT, SOX2OT-S1, and SOX2OT-S2 are coupregulated with SOX2 and OCT4 in esophageal squamous cell carcinoma. Stem Cells. 2014;32(1):126-34.

22. Yu D, Holm R, Goscinski MA, Trope CG, Nesland JM, Suo Z. Prognostic and clinicopathological significance of Cacna2d1 expression in epithelial ovarian cancers: a retrospective study. Am J Cancer Res. 2016;6(9):2088-97.

23. Sui X, Geng J, Li YH, Zhu G, Wang WH. Calcium channel a2 $\delta 1$ subunit (CACNA2D1) enhances radioresistance in cancer stem-like cells in non-small cell lung cancer cell lines. Cancer Manage Res. 2018;10:5009-18.

\section{Publisher's Note}

Springer Nature remains neutral with regard to jurisdictional claims in published maps and institutional affiliations. 